\title{
Factores que impactan en los criterios de éxito de los proyectos en Perú y Ecuador: el rol moderador de las competencias del director de proyecto
}

\author{
Washington F. Padilla ${ }^{\star}$, Ricardo M. Pino ${ }^{2}$ y Adriana A. Amaya ${ }^{3}$ \\ (1) CENTRUM Graduate Business School, Pontificia Universidad Católica del Perú, Jirón Daniel Alomía Robles 125, \\ Urbanización Los Álamos de Monterrico, Santiago de Surco 15023, Lima, Perú (correo-e: wfpadilla@pucp.edu.pe) \\ (2) Universidad Peruana de Ciencias Aplicadas, Av. Prolongación Primavera 2390, Lima 15023, Perú \\ (correo-e: ricardo.pino@upc.pe) \\ (3) Escuela Superior Politécnica del Litoral (ESPOL) - ESPAE Escuela de Negocios de la ESPOL, Calle Malecón 100 y \\ Loja, Guayaquil, Ecuador (correo-e: aamaya@espol.edu.ec) \\ * Autor a quien se debe dirigir la correspondencia
}

Recibido Ene. 11, 2021; Aceptado Mar. 13, 2021; Versión final Abr. 27, 2021, Publicado Ago. 2021

\begin{abstract}
Resumen
El objetivo de esta investigación fue examinar la relación entre los factores críticos de éxito del perfil de implementación del proyecto (PIP) con los criterios para medir el éxito del proyecto, y el grado en que varían las relaciones al ser moderadas por la experiencia, la certificación en gestión de proyectos, y el estilo de liderazgo transformacional del director de proyecto. El estudio fue no experimental, de naturaleza cuantitativa, y realizado en Perú y Ecuador para el sector de tecnologías de información (TI). Los datos fueron obtenidos por encuestas y modelados utilizando ecuaciones estructurales. Los resultados muestran que los factores críticos que explicaron el éxito fueron la aceptación del cliente, el equipo del proyecto, y la resolución de problemas. La experiencia del director de proyecto no es significativa y la certificación no incide en el éxito. Se concluye que durante el desarrollo de un proyecto es necesario combinar varios estilos de liderazgo.
\end{abstract}

Palabras clave: éxito; proyecto; factores críticos; criterios; estilo; liderazgo transformacional; certificación; experiencia

\section{Factors that affect project success criteria in Peru and Ecuador: the moderating role of the project director's competencies}

\begin{abstract}
The aim of this study was to examine the relationships between the critical success factors of the project implementation profile (PIP) with project success criteria and with the degree to which relationships vary when moderated by experience, management's project approval, and the transformational leadership style of the project's manager. This study was non-experimental, quantitative in nature, and was conducted in Peru and Ecuador in the information technology (IT) sector. Data were gathered through surveys and were modeled by using structural equations. The results showed that the critical factors that explained success were customer acceptance, project team, and problem solving. Project manager experience was not significant and certification had no relevance. It is concluded that during project development it is necessary to combine various leadership styles.
\end{abstract}

Keywords: project success; critical; factors; criteria; transformational leadership style; certification; experience 


\section{INTRODUCCIÓN}

En las últimas décadas, la gestión de proyectos como disciplina, ha ganado un notable reconocimiento. Inicialmente, esta gestión se enfocaba más al uso de técnicas de planificación y control con la finalidad de conseguir un resultado en el tiempo, presupuesto, y dentro del alcance trazado. Este rol táctico de la gestión de proyectos pasa a tener un enfoque estratégico de gestión en la organización cuando entrega productos o servicios que proporcionen valor al negocio (Müller y Jugdev, 2012). El cambio de enfoque fue impulsado por la creciente incidencia de la gestión de proyectos en los resultados esperados del negocio, al investigar la capacidad organizacional de mejorar la implementación exitosa de los proyectos (Costantino et al., 2015).

Desde diferentes perspectivas, los proyectos han sido considerados sistemas de creación de valor que contemplan no solamente el cumplimiento de restricciones duras, sino que apalancan el rendimiento del negocio basado en múltiples criterios en torno a la organización (Shenhar y Dvir, 2007). Por consiguiente, la gestión de proyectos se puede medir en términos tangibles por sus resultados financieros, así como también en términos intangibles, como son los beneficios para la cultura organizacional, la eficiencia y la satisfacción de los clientes e interesados (Zhai et al., 2009). En consecuencia, el valor de la gestión del proyecto dependerá de su implementación y del contexto organizacional donde sea desarrollado. Por consiguiente, el director de proyecto no sólo requiere contar con habilidades técnicas, sino también administrativas para gestionar las interrelaciones entre los interesados del proyecto, y desenvolverse dentro de las restricciones propias que responden a la realidad de cada organización.

Para alcanzar el valor esperado en las organizaciones por medio de los proyectos que se llevan a cabo, uno de los aspectos de mayor interés en la literatura es comprender las implicaciones del término "éxito" (Mir y Pinnington, 2014), al no representar lo mismo para todos los involucrados e interesados (Davis, 2018). Tradicionalmente el éxito se ha asociado con los resultados en el cumplimiento de los requerimientos específicos al llamado triángulo de acero (tiempo, costo y calidad), pero conforme la gestión de proyectos ha evolucionado, muchos autores han sugerido que dichos criterios no deberían ser exclusivos para evaluar si un proyecto ha sido exitoso o no (Sanchez et al., 2017). La relevancia del estudio del éxito o fracaso de los proyectos se da por el cambio de visión a lo largo de los años, enfatizando su importancia en el contexto estratégico de las organizaciones, ya que el logro de los beneficios esperados por la alta dirección en el tiempo (situación futura), dependerá del éxito de los productos o servicios generados por los proyectos.

\section{OTROS ANTECEDENTES}

La investigación de las variables que influyen en el éxito de los proyectos en las organizaciones continúa siendo de profundo interés (Gemino et al., 2020), por el impacto de la gestión de proyectos en el rendimiento de los negocios, el entorno en que se desenvuelven, y su sostenibilidad en el largo plazo. De estas variables, las competencias del director de proyecto son fundamentales para el éxito de los proyectos (Catanio et al., 2013); así como los factores de éxito, los criterios de éxito, y la relación entre ambos (Moradi et al., 2020). Los proyectos permiten a las organizaciones adaptarse a los entornos cambiantes, y alcanzar sus objetivos estratégicos, lo que resulta en niveles superiores de productividad. Adicionalmente, las organizaciones utilizan exhaustivamente proyectos y programas que, en conjunto, forman el portafolio de proyectos en los que las decisiones de inversión son tomadas en consideración.

Esta investigación aporta al conocimiento al estudiar como los factores críticos de éxito del modelo PIP de Slevin y Pinto (1986) inciden en los criterios de éxito del proyecto (Shenhar \& Dvir, 2007), y cómo se comportan las relaciones al ser moderadas por las competencias del director del proyecto (experiencia, certificación, y liderazgo) en el sector de Tecnología de la Información (TI) para Perú y Ecuador. De esta manera, es posible contribuir a ciertas brechas generadas por los resultados contradictorios en investigaciones previas. Por consiguiente, se establecen las siguientes preguntas de investigación: ¿En qué medida los factores críticos del modelo PIP influencian las dimensiones de éxito de los proyectos?, y ¿En qué medida las competencias del director de proyecto contribuyen a la relación entre los factores del modelo PIP y las dimensiones de éxito de los proyectos?

\section{Director de Proyecto}

Son varias las investigaciones que han estudiado la importancia de rol del director de proyecto en el éxito de los proyectos, y el rendimiento de las organizaciones por medio de una gestión exitosa de los proyectos. Por ejemplo, Müller y Turner (2007) encontraron que el director de proyecto es un factor decisor en el éxito o fracaso de los proyectos, sugiriendo que entre las características que debería tener un director de proyecto están la capacidad de liderazgo, las competencias adecuadas por tipo de proyecto, tener manejo de equipos, ser un buen comunicador, entre otros. Aga et al. (2016) sostuvieron que los comportamientos del director de proyecto son importantes para alcanzar un mayor éxito de los proyectos, entre los cuales, destacaron la 
influencia del estilo de liderazgo transformacional. Por otro lado, Ramos et al. (2016) analizaron la complejidad del rol como director de proyecto, mientras que Sánchez et al. (2017) enfatizaron que el éxito de la gestión del proyecto es responsabilidad del director de proyecto, y analizaron los elementos que influencian el éxito desde una perspectiva multinivel. Por tanto, se puede evidenciar que el rol del director de proyecto es crítico para el éxito del proyecto, resaltando la importancia del desarrollo de sus habilidades técnicas, de relacionamiento, y conceptuales.

\section{Factores Críticos de Éxito}

Slevin y Pinto (1986) propusieron un modelo conceptual basado en un conjunto de diez factores críticos de éxito al que denominaron Perfil de Implementación del Proyecto (PIP) para incrementar la probabilidad de éxito en los proyectos (ver Tabla 1). Su contribución es considerada seminal, y el modelo PIP fue utilizado por varios autores para postular sus aportes acerca de los factores críticos del éxito en la literatura de la gestión de proyectos (Müller y Jugdev, 2012).

Tabla 1: Factores críticos de éxito del modelo PIP (Adaptado de Slevin y Pinto, 1986)

\begin{tabular}{|l|l|}
\hline Misión del proyecto (FCMI). & Claridad de los objetivos del proyecto para todos los interesados. \\
\hline Soporte de la alta dirección (FCAD). & $\begin{array}{l}\text { Disposición del personal directivo de proveer los recursos y la autoridad } \\
\text { necesaria para el éxito del proyecto. }\end{array}$ \\
\hline Plan de proyecto (FCPL). & $\begin{array}{l}\text { Especificación detallada de las acciones requeridas para la } \\
\text { implementación del proyecto. }\end{array}$ \\
\hline Entendimiento del cliente (FCEC). & $\begin{array}{l}\text { Comunicación y consulta escuchando a todas las partes involucradas en el } \\
\text { proyecto. }\end{array}$ \\
\hline Personal del proyecto (FCPP). & $\begin{array}{l}\text { Reclutamiento, selección y capacitación del personal del equipo de } \\
\text { proyecto. }\end{array}$ \\
\hline Actividades técnicas (FCAT). & Cumplimiento de las necesidades técnicas del proyecto. \\
\hline Aceptación del cliente (FCAC). & Entrega del proyecto finalizado a satisfacción de los usuarios o clientes. \\
\hline Monitoreo y retroalimentación (FCMR). & $\begin{array}{l}\text { Entrega oportuna de la información para el control en cada etapa del } \\
\text { proyecto. }\end{array}$ \\
\hline Comunicación (FCCO). & Gestión efectiva de la información para todos los interesados del proyecto. \\
\hline Resolución de problemas (FCRP). & $\begin{array}{l}\text { Habilidad de manejar las desviaciones inesperadas durante la ejecución } \\
\text { del proyecto respecto al plan }\end{array}$ \\
\hline
\end{tabular}

Pinto y Prescott (1988) estudiaron la influencia de los factores críticos de éxito del modelo PIP (Slevin y Pinto, 1986) considerando las fases del ciclo de vida del proyecto. En general, no todos los factores fueron significativos, recalcando que la variable personal no tuvo un impacto significante sobre el éxito del proyecto. Belout y Gauvreau (2004) volvieron a analizar los factores críticos del PIP motivados por la importancia de los recursos humanos en los proyectos y las organizaciones, encontrando nuevamente que el factor personal del proyecto tampoco influyó en el éxito del proyecto, excepto para una estructura organizacional de tipo funcional. Además, no pudieron analizar el comportamiento de los factores durante la fase de finalización del proyecto debido a las características de la muestra utilizada. Investigaciones posteriores también han considerado los factores críticos de éxito del modelo PIP, como es el caso de Costantino et al. (2015), quienes comprobaron que los factores críticos de éxito "pueden servir como el criterio fundamental para prevenir posibles causas de fracasos con un efectivo proceso de selección de proyectos, tomando en cuenta los objetivos estratégicos de la compañía, la experiencia del director del proyecto, y el entorno competitivo" ( $p$. 1744).

Desde la perspectiva de la literatura de la gestión de proyectos, sigue siendo de interés identificar cuáles son aquellos factores críticos que influencian la implementación exitosa de los mismos (Jitpaiboon et al., 2019). Debido a que no hay un acuerdo acerca de qué factores influyen en el éxito, el modelo PIP contiene la lista de factores críticos de éxito más citados en la literatura (Costantino et al., 2015). A pesar que un gran número de estudios han categorizado a los factores críticos de éxito, para Davis (2018), los modelos planteados no han presentado un cambio relevante desde Pinto y Slevin (1986).

\section{Criterios de Éxito}

La investigación relacionada al éxito de los proyectos muestra que no es posible consensuar en una única lista de criterios adecuada para todos los proyectos, ya que su evaluación difiere de proyecto a proyecto y de otras características como la industria, el tamaño del proyecto, su complejidad, etc. (Müller y Turner, 2007; Shenhar et al., 2001). Esto implica aplicar varios criterios para juzgar el éxito de un proyecto, es decir, el éxito pasa a ser un concepto multidimensional. 
Shenhar et al. (2001) propusieron como dimensiones para describir el éxito del proyecto a la eficiencia, impacto en el cliente, éxito del negocio, y preparación para el futuro de la organización. Por otro lado, Belout y Gauvreau (2004) analizaron a la gestión del recurso humano como uno de los elementos principales que influyen en el éxito de los proyectos. Posteriormente, Shenhar y Dvir (2007) añadieron el impacto sobre el equipo del proyecto (ver Tabla 2). El análisis de la literatura acerca de los criterios de éxito del proyecto, pone en evidencia la necesidad de medir el éxito desde un enfoque más integral, contemplando los puntos de vista de los interesados principales del proyecto.

Tabla 2: Criterios de éxito (Adaptado de Shenhar y Dvir, 2007)

\begin{tabular}{|l|l|}
\hline Eficiencia Misión del proyecto & Cumplimiento de las metas planificadas (cronograma, costo, alcance). \\
\hline Impacto en el cliente. & $\begin{array}{l}\text { Direccionamiento de las necesidades del cliente por medio de los resultados del } \\
\text { proyecto. }\end{array}$ \\
\hline Impacto en el equipo. & Medición de como el proyecto afecta al equipo y sus miembros. \\
\hline Éxito del negocio. & Impacto directo del proyecto a la organización. \\
\hline Preparación para el futuro. & Releja si el proyecto ayuda a la organización a aprovechar futuras oportunidades. \\
\hline
\end{tabular}

\section{Relación Factores Críticos de Éxito y Criterios de Éxito}

En la literatura en gestión de proyectos, se hace referencia con frecuencia a los factores de éxito del proyecto y los criterios de éxito del proyecto como componentes principales del éxito de los proyectos, siendo Pinto, Slevin y Prescott pioneros en este campo (Müller y Jugdev, 2012). Si se toma en cuenta el sector o la industria, la importancia de los factores para el éxito de los proyectos varía según las fases del ciclo de vida y el tipo de proyecto (Belout y Gauvreau, 2004). Es decir, un mismo conjunto de variables no afecta del mismo modo al éxito de todos los proyectos, generando así la necesidad de identificar los factores que son críticos a cada proyecto (Jitpaiboon et al., 2019). Mir y Pinnington (2014), concluyeron que hay una relación general e individual entre las variables de rendimiento de la gestión del proyecto con las dimensiones de éxito del proyecto (Shenhar et al., 2001). Serrador y Turner (2015), por su parte, utilizaron las dimensiones de éxito del proyecto propuestas por Shenhar y Dvir (2007) para demostrar que la eficiencia del proyecto contribuye de manera significativa al éxito del proyecto. A pesar de los numerosos estudios que analizan los factores que llevan al éxito de los proyectos, para Osei-Kyei et al. (2017), la relación entre factores críticos de éxito y criterios de éxito no ha sido todavía suficientemente investigada.

La literatura sugiere que un director de proyecto debe cuidar la calidad de su gestión para influenciar positivamente en el desarrollo de las actividades del proyecto durante todo su ciclo de vida (inicio, planificación, ejecución, y cierre). De esta forma los entregables generados por el equipo del proyecto, ya sea para un producto o servicio, deben cumplir con las expectativas de los interesados, y así el proyecto pueda ser catalogado como exitoso o fallido. Esto implica identificar cuáles son los factores críticos de éxito que permiten dirigir eficientemente un proyecto (Costantino et al., 2015), tomando en cuenta que la relevancia de los factores puede diferir entre industrias (Belout y Gauvreau, 2004). Considerando que en el sector de TI, se presenta un importante índice de proyectos que no cumplen con las condiciones iniciales, se consideró pertinente examinar la relación entre los diez factores críticos de éxito del modelo PIP (Slevin y Pinto, 1986) con el éxito del proyecto bajo el enfoque multidimensional propuesto por Shenhar y Dvir (2007) para proyectos que hayan sido finalizados. De esta manera, es posible identificar cuál es el conjunto de factores que permitan satisfacer el cumplimiento de los requisitos del proyecto. Se plantea entonces la siguiente hipótesis:H1: Los factores críticos de éxito del modelo PIP influencian positivamente sobre los criterios de éxito del proyecto.

\section{Competencias del Director de Proyecto}

El desarrollo de las competencias en las organizaciones es considerado como un factor de éxito para alcanzar una ventaja competitiva (Medina y Medina, 2014), y en gestión de proyectos, el desarrollo de las competencias del individuo que ejerce el rol de director de proyecto es clave para la efectividad en su gestión. De las competencias que debe desarrollar el director de proyecto, la experiencia y conocimiento son competencias relevantes para el director de proyecto que influencian la toma de decisiones, incrementando las oportunidades de éxito del proyecto (Catanio et al., 2013).

De acuerdo a Hoxha y McMahan (2018), hay un consenso de que la experiencia del director de proyecto influye en su proceso de contratación. Según Savelsbergh et al. (2016), la experiencia se relaciona con la práctica, y aunque es esencial para el aprendizaje del director de proyecto, un mayor tiempo de experiencia no necesariamente implica una mayor efectividad en la gestión de proyectos (Medina y Medina, 2014). Hay varios estudios la literatura que han encontrado una correlación significativa entre la experiencia del director 
del proyecto y el éxito del proyecto (Müller y Turner, 2007) o simplemente ninguna significancia (Hoxha y McMahan, 2018). Desde el punto de vista organizacional, la experiencia del recurso humano está relacionado con su rendimiento, además de ser considerada un requisito indispensable para el proceso de decisión y asignación de proyectos en las organizaciones (Hoxha y McMahan, 2018). Una de las aristas del aprendizaje se relaciona con el nivel de experiencia del director de proyecto en el cumplimiento de su rol (Savelsbergh et al., 2016), incidiendo en la forma que el director de proyecto aborda los factores críticos de éxito para la efectividad de la gestión del proyecto, y la gestión de los interesados a los cuales va dirigido el proyecto. La experiencia ha sido tomada en cuenta en esta investigación por su relación con la capacidad del director de proyecto para su entrega exitosa de acuerdo a las expectativas de los interesados, de este modo, se propone la siguiente hipótesis: H2a: La competencia del director de proyecto, que incluye la experiencia en gestión de proyectos, influencia positivamente en la relación entre los factores del modelo de perfil de implementación del proyecto (PIP) y los criterios de éxito de los proyectos.

El conocimiento ayuda a formar las competencias del director de proyecto (Savelsbergh et al., 2016), y se lo adquiere a través de varias fuentes. Las asociaciones profesionales de gestión de proyectos como el Project Management Institute (PMI), difunden las mejores prácticas en el campo, y otorgan certificaciones profesionales que reflejan el conocimiento y experiencia adquirida por los directores de proyectos. Al respecto, Savelsbergh et al. (2016) dijeron lo siguiente "...no sabemos cuáles experiencias y por qué estas experiencias Ilevaron a los autores del PMI a concluir que sus lecciones aprendidas son realmente las mejores prácticas" (p. 560). Pese al crecimiento vertiginoso de certificaciones a nivel mundial, aún no se cuenta con suficiente evidencia de los beneficios de contar o no con una certificación, y el valor que es entregado a las organizaciones, sin embargo, representan un criterio importante a favor de la selección de personal calificado en gestión de proyectos por parte de las organizaciones (Catanio et al., 2013). Por ejemplo, Müller y Turner (2007) encontraron que, solo para proyectos de alto rendimiento, los líderes certificados incidían en el éxito de los proyectos más que aquellos que no contaban con una certificación. Para Catanio et al. (2013), la certificación es apenas una pequeña parte de la dimensión del conocimiento, como para considerar que incrementa la probabilidad de éxito de los proyectos.

En esta línea de investigación, Joseph y Marnewick (2017) concluyeron que una certificación en gestión de proyectos, por sí sola, no implica que un director de proyecto lo gestionará exitosamente. Obtener una credencial reconocida internacionalmente en gestión de proyectos es bien vista por los practicantes el campo, ya que se esperan niveles superiores en el rendimiento de un director de proyecto certificado respecto a un director de proyecto no certificado, por la profundidad en el conocimiento de las mejores prácticas en gestión de proyectos, lo que implica un mayor entendimiento del impacto de los factores críticos de éxito sobre el éxito del proyecto. Debido a que las organizaciones realizan inversiones importantes en directores de proyecto certificados, en especial el sector de TI (Joseph y Marnewick, 2017), se propone la siguiente hipótesis: H2b: La competencia del director de proyecto, que incluye la credencial de una certificación otorgada por el PMI, influencia positivamente en la relación entre los factores del modelo de perfil de implementación del proyecto (PIP) y los criterios de éxito de los proyectos.

Además de la experiencia y el conocimiento, la literatura en gestión de proyectos destaca la competencia de liderazgo del director de proyecto para influenciar en el rendimiento de los miembros de su equipo y en la construcción de relaciones con los distintos interesados a favor del proyecto. Entre los estilos de liderazgo, el estilo de liderazgo transformacional es considerado como un fuerte predictor para el éxito del proyecto (Aga et al., 2016; Tyssen et al., 2014), por su capacidad motivadora e inspiradora, que se traduce en una mejor toma de decisiones dentro del contexto en el que se gestionan los proyectos, afectando positivamente el desarrollo de las actividades de los proyectos hasta su entrega exitosa. El liderazgo transformacional puede verse afectado por las características propias del proyecto, la estructura organizacional, y la naturaleza temporal de los proyectos (Tyssen et al., 2014). Al considerar el contexto en el proyecto, el liderazgo transformacional también está relacionado, positivamente, a la construcción de un equipo comprometido con el proyecto al establecer claramente los objetivos, clarificar roles, potenciar las relaciones interpersonales y la capacidad de resolución de problemas (Aga et al., 2016). En base a esto, se plantea la siguiente hipótesis: H2c: La competencia del director de proyecto, que incluye el estilo de liderazgo transformacional, influencia positivamente en la relación entre los factores del modelo de perfil de implementación del proyecto (PIP) y los criterios de éxito de los proyectos.

Acorde al marco teórico, en el presente estudio se consideran como variables exógenas a los factores críticos de éxito del modelo PIP para la gestión del proyecto; como variable endógena a los criterios de éxito del proyecto para catalogar si fue exitoso o no y, como variable moderadora la competencia del director del proyecto, medida por la experiencia, posesión de una certificación profesional, y por el estilo de liderazgo transformacional. La Figura 1 ilustra lo explicado en el marco teórico. 


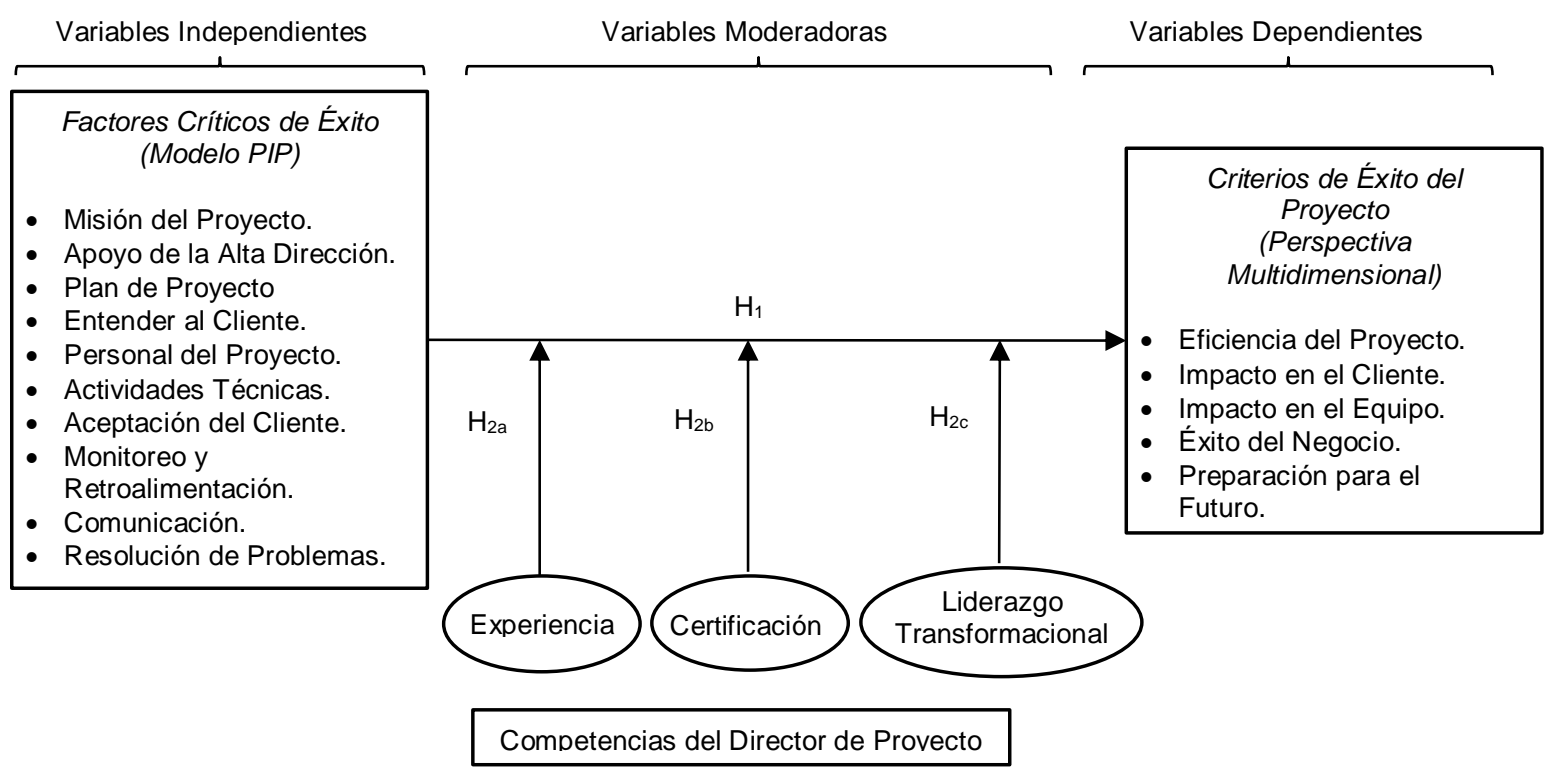

Fig. 1: Marco teórico

\section{METODOLOGÍA}

Al ser una investigación de naturaleza cuantitativa se busca estudiar y medir cómo cambian las variables, con el fin de describir su comportamiento mediante un proceso de análisis deductivo. Todas las variables de la investigación fueron medidas utilizando múltiples indicadores, con excepción de la experiencia y posesión de la certificación profesional otorgada por el PMI. Los 10 factores críticos de éxito del modelo PIP se midieron por medio del instrumento propuesto por Slevin y Pinto (1986) que consta de 50 preguntas (cinco preguntas por cada factor). Las dimensiones o criterios para evaluar el éxito del proyecto fueron medidos con el instrumento propuesto por Shenhar y Dvir (2007), que consta de 20 preguntas divididas en: cuatro preguntas para la eficiencia del proyecto, cinco preguntas para el impacto sobre el cliente/usuario, tres preguntas para el impacto sobre el equipo del proyecto, cuatro preguntas sobre el éxito del negocio, y cuatro preguntas sobre la preparación de la organización para el futuro. Para medir el estilo de liderazgo transformacional del director de proyecto se utilizó el instrumento adaptado por Aga et al. (2016) que consta de 13 preguntas. Finalmente, para medir la experiencia del director de proyecto, y si cuenta con una certificación en gestión de proyectos otorgada por el PMI, se utilizaron dos preguntas (Müller y Turner, 2007).

\section{Población}

El estudio se realizó en el contexto latinoamericano, específicamente en Perú y Ecuador. La población está conformada por individuos que han ejercido el rol de directores de proyectos en organizaciones de la industria de TI establecidas en estos países. Los directores de proyectos objeto del estudio no necesariamente tuvieron una posición jerárquica dentro de sus organizaciones, pero se consideró su experiencia dirigiendo proyectos, así como la posesión o no de una certificación profesional internacional de gestión de proyectos emitida por el PMI que avale su conocimiento. Los individuos seleccionados para la participación en el estudio fueron contactados vía correo electrónico y de manera presencial.

\section{Marco Muestral}

La muestra seleccionada es de naturaleza no probabilística, establecida mediante el método de muestreo por conveniencia, haciendo partícipes a los individuos que cumplieron con las características requeridas para la investigación. El muestreo por conveniencia es un procedimiento muy útil, razón por la cual es común su uso en el campo de la investigación para los negocios y la administración. Para el criterio de selección de la muestra se consideró lo siguiente (Dörnyei, 2007): (a) individuos de la población con la posición, conocimiento, y experiencia para poder responder adecuadamente las preguntas de la encuesta; (b) proximidad geográfica; (c) disponibilidad de tiempo para responder la encuesta; y (d) accesibilidad o voluntad de responder la encuesta. Debido a que el muestreo por conveniencia puede proveer buenos resultados o no, como medidas para controlar la incertidumbre y el sesgo se cuidó la representatividad de la muestra, se distribuyó la encuesta en distintos momentos de tiempo, y se incorporó más datos a la muestra. La encuesta fue enviada a más de 1,000 individuos, quienes fueron contactados a través de la red social Linkedln y los capítulos locales de Lima y Quito del PMI. Se enfatizó que los proyectos a ser evaluados por los directores de proyectos debían haber finalizado en un período no mayor a un año desde la fecha de participación para la presente investigación. 
El número total de respuestas recibidas fue de 324 encuestas, que luego de un proceso de depuración quedaron 211 encuestas válidas, mayor a las 131 observaciones obtenidas por medio del software G*Power 3.1.9.4, considerando un nivel de significancia estadística $(\alpha)$ del $5 \%$, nivel de potencia $(1-\beta)$ de .80, y tamaño del efecto de Cohen $\left(\mathrm{f}^{2}\right)$ de .15 (Hair et al., 2019).

\section{Adecuación Muestral.}

Para la medición de la adecuación muestral, y determinar si es apropiado llevar a cabo el análisis factorial, se utilizó la medida de Kaiser-Meyer-Olkin (MKO) y la prueba de esfericidad de Bartlett para determinar la significancia de las correlaciones entre las variables. Los resultados del índice KMO y de la prueba de Bartlett muestran que las correlaciones entre pares de variables pueden ser explicadas por otras variables $(\mathrm{KMO}=$ .868), y debido a que la fiabilidad fue inferior a .05 se rechaza la hipótesis nula de que las variables no están correlacionadas en la población.

\section{Validez del Instrumento}

Para determinar si el instrumento de medición mide lo que se espera sobre una base de confiabilidad, en primer lugar, el contenido del instrumento fue validado mediante la ejecución de una prueba piloto con un grupo de directores de proyectos seleccionados para asegurar el entendimiento del mismo. Posteriormente el cuestionario fue refinado para redactar las preguntas de acuerdo a los resultados de esta prueba, e incrementar la comprensión de las preguntas por parte de los encuestados. Por último, se aplicó la prueba de validación del instrumento por medio de la validez de criterio, para lo cual se correlacionó cada variable independiente con los criterios de éxito del proyecto, mostrando que el modelo tiene una validez de criterio, ya que para el tamaño de la muestra de 211 observaciones los coeficientes de correlación excedieron el valor crítico de .135 .

\section{Varianza del Método Común (CMV)}

Al medir variables latentes indirectamente de manera simultánea, a través de un mismo instrumento, se pueden presentar sesgos que alteren las respuestas del cuestionario de la investigación, lo que provoca la presencia de la varianza del método común (CMV). Esto podría llevar a errores tipo I (falso positivos) o tipo II (falso negativos). Para controlar la CMV, se siguieron las recomendaciones de Podsakoff et al. (2003), orientadas al procedimiento de la investigación y manteniendo un nivel estadístico. A nivel de procedimiento, en el cuestionario se separaron las variables independientes y dependientes, se cuidó la comprensión del instrumento redactando las preguntas de la manera más directa posible, así como se garantizó el anonimato de los encuestados, y se obtuvieron las respuestas en distintos momentos del tiempo. A nivel estadístico, se aplicó la prueba del factor único de Harman (Hair et al., 2019), obteniendo un valor de $26.30 \%$, inferior al $40 \%$ recomendado, lo que sugiere que la CMV no es una amenaza para los datos de la muestra.

\section{Análisis de los Datos}

Para el análisis de los datos se utilizó la modelación por ecuaciones estructurales (SEM) con enfoque por mínimos cuadrados parciales (PLS). El modelo PLS está definido por el modelo externo (relaciones entre la variable latente y sus indicadores observados) y el modelo estructural (relaciones entre variables latentes). Para la evaluación del modelo de medición externo se comprobó (Hair et al., 2019): (a) la fiabilidad de consistencia interna con un rango de valores entre .768 a .912 para los coeficientes alfa de Cronbach, mayores al valor de .70 recomendado; (b) la fiabilidad de los indicadores observables comprobando cargas superiores a .60; y (c) la validez convergente por medio de la varianza promedio extraída (AVE), tomando como criterio un valor mayor .40; (d) la validez discriminante verificando que los estimados AVE son mayores a las correlaciones entre los otros constructos del modelo. Para la evaluación del modelo estructural o modelo interno, se consideró: (a) la evaluación de la multicolinealidad entre los constructos por ser un modelo reflectante, verificando que el factor de inflación de la varianza (VIF) no supere el valor de 5.0; (b) la determinación de los coeficientes de las relaciones entre variables latentes; (c) la evaluación del valor crítico t de Student; (d) la estimación del coeficiente de determinación $\left(R^{2}\right)$; (e) la estimación del tamaño del efecto de Cohen $\left(\mathrm{f}^{2}\right)$; y (f) la estimación de la relevancia predictiva $\left(\mathrm{Q}^{2}\right)$.

\section{RESULTADOS Y DISCUSIÓN}

El uso de PLS-SEM en la presente investigación se sustentó por el cumplimiento de los siguientes elementos (Hair et al., 2019; Reinartz et al., 2009): (a) complejidad del modelo estructural propuesto (enfoque predictivo) por la cantidad de relaciones al incluir varios constructos y/o indicadores; (b) los datos de la muestra no están normalmente distribuidos; (c) a pesar de que PLS se relaciona con uso de modelos de medición formativa, maneja tanto medidas reflectivas como formativas; $y(d)$ este enfoque es preferible para muestras menores a 250 observaciones. Para la aplicación de la modelación por ecuaciones estructurales con el enfoque PLSSEM se utilizó el paquete computacional SmartPLS v.3.2.8. Se realizó el análisis en dos etapas: análisis del modelo entre los factores críticos de éxito del instrumento PIP y los criterios de éxito del proyecto como 
constructo de orden superior; y análisis del modelo incluyendo el rol moderador del director de proyecto para cada una de las competencias (experiencia, certificación, y liderazgo transformacional).

\section{Evaluación del Modelo sin Variables Moderadoras}

El análisis inició incluyendo todas las variables exógenas del modelo de investigación relacionándose de manera individual con los criterios de éxito del proyecto, con la finalidad de determinar si los factores contribuyeron al éxito de los proyectos o cuál sería la combinación de factores que influenciaron el éxito de los proyectos. Los criterios de éxito del proyecto, al ser un constructo de segundo orden, contienen todos los indicadores de las dimensiones de éxito que lo componen (Shenhar y Dvir, 2007). Confirmada la validez y fiabilidad del modelo, se procedió a medir los resultados del modelo interno y contrastar las hipótesis, observando su relevancia predictiva y las relaciones entre los constructos. Para realizar la inferencia estadística, PLS-SEM requiere del proceso de Bootstrapp no paramétrico para examinar la capacidad predictiva del modelo y las relaciones entre los constructos del modelo interno (5,000 submuestras). El tipo de prueba para el bootstrapping fue de una cola por la direccionalidad de las hipótesis planteadas.

La capacidad predictiva del modelo representado por $\mathrm{R}^{2}$, indica que los factores críticos del instrumento PIP explican el $38.95 \%$ de la varianza en los criterios de éxito. Por el intervalo de confianza del tamaño del efecto $\mathrm{f}^{2}$ de las variables latentes predictivas, los factores críticos aceptación del cliente y comunicación pueden tener un efecto débil a moderado, y personal de proyecto y resolución de problemas un efecto moderado sobre los criterios de éxito del proyecto. Por último, el valor de $Q^{2}$ para esta investigación fue de .151, lo cual representa una relevancia predictiva moderada para el constructo endógeno criterios de éxito. Por los resultados mostrados en la Tabla 3 , la hipótesis $\mathrm{H}_{1}$ es soportada para el siguiente conjunto de factores críticos de éxito: (a) Aceptación del Cliente $(p<.05)$, (b) Personal del Proyecto o equipo del proyecto $(p<.01)$, y (c) la capacidad de Resolución de Problemas $(p<.01)$. De estos factores, Slevin y Pinto (1986) resaltaron la importancia de la comunicación y la resolución de problemas cuando propusieron el instrumento PIP.

Tabla 3: Significancia de las rutas del modelo interno. ${ }^{*} p<.05 ;{ }^{* *} p<.01$ (Prueba a una cola)

\begin{tabular}{|l|c|c|c|l|}
\hline Camino Hipotético & Betas & Estadístico-t & Valor- $p$ & Inferencia \\
\hline Aceptación Cliente -> Criterios Éxito & .190 & 2.038 & $.021^{*}$ & Significante \\
\hline Actividades Técnicas -> Criterios Éxito & -.060 & .589 & .278 & No-significante \\
\hline Apoyo AD -> Criterios Éxito & .005 & .066 & .474 & No-Significante \\
\hline Comunicación -> Criterios Éxito & -.126 & 1.401 & .081 & No-Significante \\
\hline Entendimiento Cliente -> Criterios Éxito & -.005 & .065 & .474 & No-significante \\
\hline Misión -> Criterios Éxito & .150 & .909 & .182 & No-significante \\
\hline Monitoreo y Retroalimentación -> Criterios Éxito & .056 & .589 & .278 & No-significante \\
\hline Personal Proyecto -> Criterios Éxito & .257 & 2.814 & $.002^{* *}$ & Significante \\
\hline Plan Proyecto -> Criterios Éxito & .045 & .473 & .318 & No-significante \\
\hline Resolución Problemas -> Criterios Éxito & .253 & 2.931 & $.002^{* *}$ & Significante \\
\hline
\end{tabular}

La débil significancia de la comunicación y su relación negativa con el éxito del proyecto fue un hallazgo no esperado y contradictorio con otras investigaciones que evidencian la importancia de la comunicación para el éxito de los proyectos. Esto pudiera responder al entorno en que se desarrollaron los proyectos, donde el director de proyecto podría depender de la gestión de las relaciones dentro de la estructura jerárquica de las organizaciones (Ramos et al., 2016), afectando la calidad de la comunicación. Otro factor que pudo haber incidido en el resultado, es posiblemente el énfasis del director de proyecto en su gestión hacia los aspectos técnicos del proyecto, descuidando la comunicación con los diferentes interesados (Mir y Pinnington, 2014). Este hallazgo también podría tener una explicación como consecuencia del tipo de comunicación utilizado, y/o la manera en que la información fue transmitida. La estructura de comunicación en un proyecto contempla el uso de canales formales e informales, no obstante, el uso de una comunicación de tipo informal podría relacionarse negativamente con el éxito del proyecto al generar escenarios donde el mensaje puede llegar distorsionado u ocasionar conflictos entre los miembros del equipo (Wu et al., 2017).

\section{Efecto Moderador de la Experiencia}

El efecto de la experiencia del director de proyecto sobre las relaciones entre los constructos del instrumento PIP y los criterios de éxito fue el siguiente (ver Tabla 4): (a) Misión $(p<.05)$, (b) Personal del Proyecto $(p<$ $.01)$, (c) Resolución de Problemas $(p<.01), y$ (d) las interacciones de la experiencia con el Entendimiento del Cliente $(p<.05)$ y la Resolución de Problemas $(p<.01)$. El valor del coeficiente de determinación $R^{2}$ representó el $43.86 \%$ de la varianza explicada de los criterios de éxito del proyecto $\left(\Delta R^{2}=12.61 \%\right)$. La magnitud de las relaciones se reflejó a través del tamaño del efecto $\mathrm{f}^{2}$ y los intervalos de confianza relacionados. En cuanto a la relevancia predictiva $\mathrm{Q}^{2}$, se registró un efecto moderado de .167 en el constructo de orden superior de las dimensiones de los criterios de éxito, superior al valor de .151 obtenido en el modelo sin la presencia de las competencias del director de proyecto. 
Tabla 4: Significancia de las relaciones entre constructos (Experiencia como moderadora). ${ }^{*} \mathrm{p}<.05 ;{ }^{* *} \mathrm{p}<.01$ (Prueba a una cola)

\begin{tabular}{|l|c|c|c|c|}
\hline Camino Hipotético & Betas & Estadístico-t & Valor- $p$ & Inferencia \\
\hline Comunicación -> Criterios Éxito & -.144 & 1.498 & .067 & No-significante \\
\hline EC_Exp_CE -> Criterios Éxito & -.168 & 1.876 & $.030^{\star}$ & Significante \\
\hline Entendimiento Cliente -> Criterios Éxito & -.010 & .102 & .459 & No-significante \\
\hline Experiencia -> Criterios Éxito & -.028 & .411 & .340 & No-significante \\
\hline Misión -> Criterios Éxito & .247 & 2.239 & $.013^{\star}$ & Significante \\
\hline Personal Proyecto -> Criterios Éxito & .265 & 2.947 & $.002^{\star \star}$ & Significante \\
\hline RP_Exp_CE -> Criterios Éxito & .231 & 2.900 & $.002^{\star *}$ & Significante \\
\hline Resolución Problemas -> Criterios Éxito & .268 & 2.860 & $.002^{\star *}$ & Significante \\
\hline
\end{tabular}

En esta investigación la experiencia no fue significativa, lo que podría ser consecuencia de una confusión no intencionada por parte de los encuestados entre experiencia participando en proyectos y experiencia dirigiendo proyectos. Las respuestas de los encuestados también pudieron verse afectadas por la experiencia previa en proyectos no relacionados a TI, o el nivel de aprendizaje adquirido por el grado de involucramiento en los proyectos que tuvieron participación. Los resultados sugieren que las habilidades duras de los directores de proyecto que participaron en la investigación primaron sobre las habilidades blandas, ya que, por el comportamiento de la moderación, no se comprendió adecuadamente las necesidades del cliente, pero si apalancó la capacidad de resolución de problemas del equipo de proyecto, en beneficio del éxito del proyecto.

Con la experiencia como moderadora, se mantuvo la no-significancia de la comunicación, pero mejoró el valor-p respecto al registro sin moderación. Este resultado es distinto a lo obtenido por Pinto y Prescott (1988) y Belout y Gauvreau (2004), quienes, a pesar de reconocer la importancia de la comunicación para la implementación exitosa de un proyecto, por el comportamiento de los datos recolectados, no pudieron incluir este factor en su análisis. Otro hallazgo para resaltar fue que la relación entre el factor crítico misión y los criterios de éxito se volvió positivamente significativa, lo que refleja la importancia para la gestión del proyecto al establecer claramente desde el inicio cuáles son los objetivos y beneficios para alcanzar, con lo cual, se entrega en el cierre del proyecto el producto o servicio esperado por el cliente y/o usuario.

En relación con el factor crítico de éxito entendimiento del cliente, Pinto y Prescott (1988) lo identificaron como un factor significativo en las fases conceptual, ejecución, y finalización del proyecto. La interacción refleja un esfuerzo del director de proyecto por definir el producto o servicio lo más temprano posible de acuerdo a las necesidades del cliente/usuario, ya que una debilidad en este factor puede llevar a un concepto erróneo de lo que debe generar el proyecto. En cuanto al factor crítico de éxito resolución de problemas, Pinto y Prescott (1988) lo encontraron significativo durante la fase de ejecución del proyecto. En la misma línea, Belout y Gauvreau (2004) en todas las etapas del proyecto, lo correlacionaron significativamente con los proyectos llevados a cabo en el sector de TI.

La consideración de la experiencia como variable moderadora soportó parcialmente la hipótesis $\mathrm{H}_{2 \mathrm{a}}$, específicamente para los siguientes casos: (a) relación del factor crítico Entendimiento del Cliente con los criterios de éxito del proyecto (ver Figura 2a), sugiriendo que si el nivel de entendimiento de las necesidades del cliente es alto, se obtienen mejores resultados con un nivel bajo de experiencia del director de proyecto; y (b) relación del factor crítico Resolución de Problemas con los criterios de éxito del proyecto (ver Figura 2b), evidenciando que en el contexto de una alta experiencia del director de proyecto, a un alto nivel de capacidad de resolución de problemas por parte del equipo de proyecto, el éxito del proyecto será mayor.

\section{Efecto Moderador de la Certificación}

Los factores que explicaron los criterios de éxito del proyecto fueron (ver Tabla 5): (a) Certificación $(p<.05)$, (b) Misión ( $p<.05)$, (c) Personal del Proyecto $(p<.05)$, (d) Resolución de Problemas $(p<.01)$ y (e) las interacciones de la certificación con el Entendimiento del Cliente y la Misión $(p<.05)$. La varianza explicada o $R^{2}$ para los criterios de éxito fue de $45.47 \%\left(\Delta R^{2}=16.75 \%\right)$, y la relevancia predictiva $Q^{2}$ registró un efecto moderado de .174.

Los resultados muestran que la inclusión de la certificación como variable moderadora soportó parcialmente la hipótesis $\mathrm{H}_{2 b}$. El primer hallazgo importante del presente análisis fue la significancia de la relación entre la certificación y los criterios de éxito del proyecto de TI en todas sus dimensiones. Esto aporta a la discusión ya que en la literatura no se tienen resultados concluyentes de la relación de la certificación con el éxito de los proyectos. Al incluir la certificación como moderadora, la misión también afectó positivamente al éxito del proyecto, lo cual está en concordancia con los hallazgos presentados en estudios anteriores. 
a)

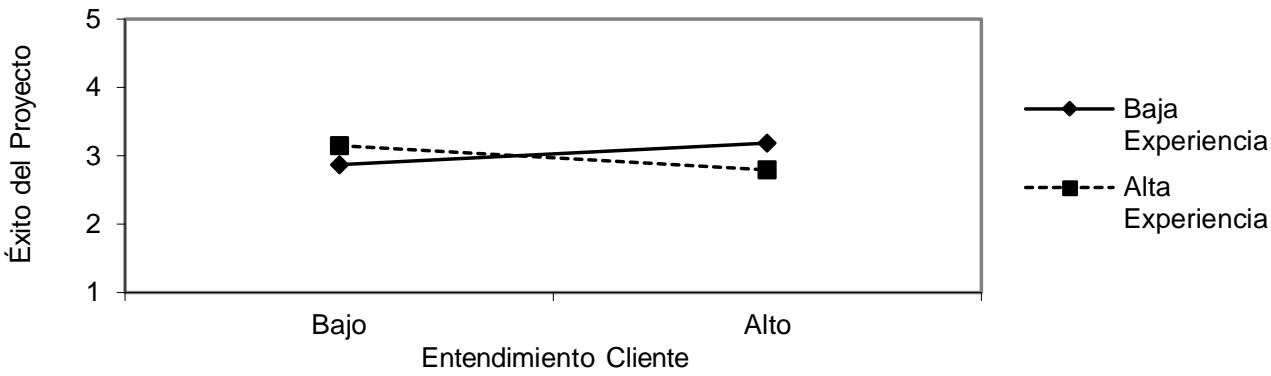

b)

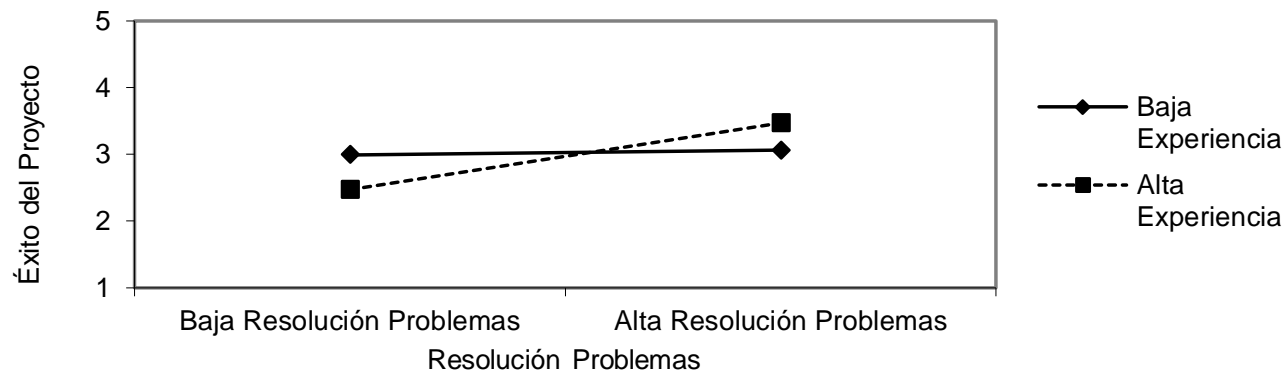

Fig. 2. Interacción: a) del entendimiento al cliente y la experiencia; y b) resolución de problemas y la experiencia

Para Pinto y Prescott (1988) el factor crítico de la misión fue significativo con el éxito en todas las fases del ciclo de vida del proyecto, y para Belout y Gauvreau (2004) la misión fue significativa en la fase de planificación del proyecto.

Tabla 5: Significancia de las relaciones entre constructos (Certificación como moderadora). ${ }^{*} p<.05 ;{ }^{* *} p<.01$ (Prueba a una cola)

\begin{tabular}{|l|c|c|c|c|}
\hline Camino Hipotético & Beta & Estadístico-t & Valor- $p$ & Inferencia \\
\hline Certificación -> Criterios Éxito & -.111 & 2.062 & $.020^{*}$ & Significante \\
\hline Comunicación -> Criterios Éxito & -.160 & 1.586 & .056 & No-significante \\
\hline EC_Cert_CE -> Criterios Éxito & -.143 & 1.971 & $.024^{*}$ & Significante \\
\hline MI_Cert_CE -> Criterios Éxito & .312 & 2.097 & $.018^{*}$ & Significante \\
\hline Misión -> Criterios Éxito & .269 & 2.061 & $.020^{*}$ & Significante \\
\hline Personal Proyecto -> Criterios Éxito & .191 & 2.139 & $.016^{*}$ & Significante \\
\hline Resolución Problemas -> Criterios Éxito & .250 & 2.937 & $.002^{\star \star}$ & Significante \\
\hline
\end{tabular}

La comunicación también fue no-significativa, asociada negativamente con el éxito, y con un mejor registro del valor-p que con la experiencia como moderadora. La certificación actuó como variable moderadora que explica la heterogeneidad en la relación para los siguientes casos: (a) relación entre la Misión y los criterios de éxito del proyecto (ver Figura 3a), evidenciando que mientras mejor definida sea la misión de un proyecto, el éxito es superior con directores de proyectos certificados; y (b) significancia de la interacción del Entendimiento del Cliente con la certificación (ver Figura 3b). En sí, distintos niveles de entendimiento del cliente prácticamente no hay diferencia en el rendimiento del proyecto cuando es dirigido por un director no certificado.

Estos resultados evidencian que un director de proyecto certificado no necesariamente aporta al éxito del proyecto. A pesar de la significancia de la certificación, conforme al análisis realizado, no hubo una diferencia significativa para el rendimiento del proyecto entre directores certificados de los no certificados. Estos hallazgos están alineados con Catanio et al. (2013), que indicaron que una certificación no significa mayor efectividad en el proyecto. Así mismo, Joseph y Marnewick (2017) resaltaron que mantener una certificación no es mandatorio para el éxito esperado de un proyecto.

La certificación en gestión de proyectos ha mantenido un crecimiento vertiginoso en los últimos años, por lo que merece un análisis especial por sus implicaciones a nivel académico y profesional. Las organizaciones generalmente reclutan individuos con experiencia en gestión de proyectos, de preferencia certificados, ya que la credencial garantizaría niveles superiores en el rendimiento de los proyectos bajo su gestión respecto a directores de proyectos no certificados. 
a)

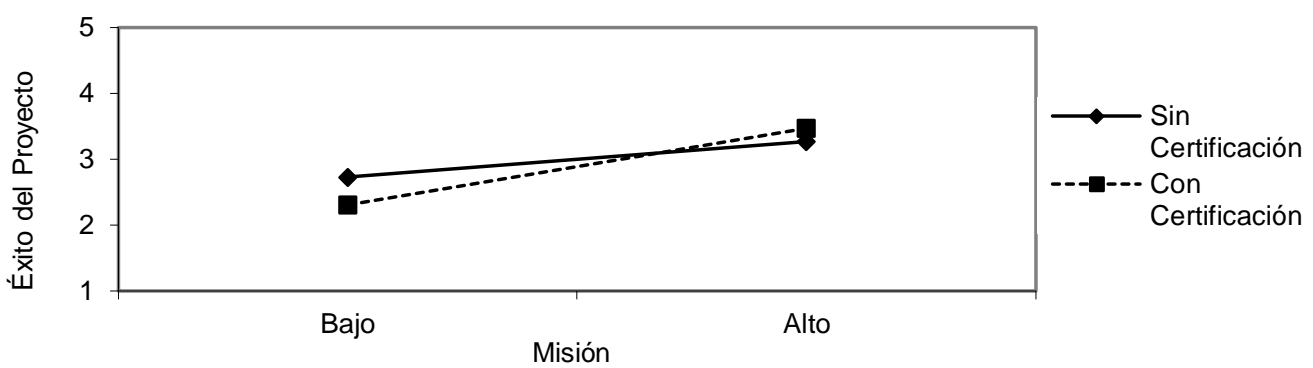

b)

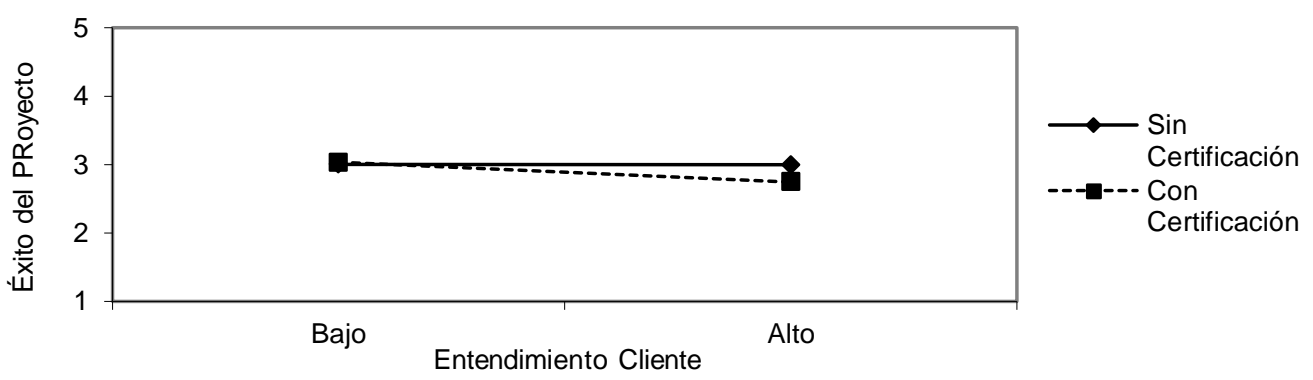

Fig. 3. Interacción: a) de misión y la experiencia; y b) del entendimiento al cliente y la experiencia

Desde la perspectiva de las universidades e institutos de capacitación, una de las propuestas de valor en sus programas académicos es que los estudiantes puedan acceder a la preparación para obtener una determinada credencial. Esta investigación evidencia una realidad adversa a lo que organizaciones y universidades esperan, ya que mostró que un director de proyecto certificado no asegura el éxito del proyecto, y no marca una diferencia positiva de un director de proyecto no certificado. En consecuencia, hay que cuestionar qué tanto una certificación es el reflejo real de la experiencia, conocimiento, y habilidades de un director de proyecto.

\section{Efecto Moderador del Liderazgo Transformacional}

Una vez realizado el proceso de bootstrapping, el porcentaje de la varianza explicada $R^{2}$ fue $47.62 \%\left(\Delta R^{2}=\right.$ $22.26 \%$ ), el estilo liderazgo transformacional tuvo un efecto significativo sobre los criterios de éxito del proyecto, y la relevancia predictiva $\mathrm{Q}^{2}$ registró un efecto moderado de .185 . Los constructos que afectaron a los criterios de éxito fueron (ver Tabla 6): (a) Liderazgo Transformacional $(p<.01)$, (b) Personal del Proyecto $(p<.05)$, (c) Resolución de Problemas $(p<.05)$, y (d) la moderación del liderazgo transformacional con el Monitoreo y Retroalimentación y con el Personal del Proyecto $(p<.05)$.

Tabla 6: Significancia de las relaciones entre constructos (Liderazgo

Transformacional como moderadora). ${ }^{*} p<.05 ;{ }^{* *} p<.01$ (Prueba a una cola)

\begin{tabular}{|l|c|c|c|c|}
\hline Camino Hipotético & Beta & Estadístico-t & Valor- $p$ & Inferencia \\
\hline Lid_Transformacional -> Criterios Éxito & .290 & 4.229 & $.000^{\star *}$ & Significante \\
\hline MR_LTransf_CE -> Criterios Éxito & .206 & 2.032 & $.021^{\star}$ & Significante \\
\hline PP_LTransf_CE -> Criterios Éxito & -.114 & 1.738 & $.041^{*}$ & Significante \\
\hline Personal Proyecto -> Criterios Éxito & .169 & 2.180 & $.015^{\star}$ & Significante \\
\hline Resolución Problemas -> Criterios Éxito & .168 & 2.032 & $.021^{*}$ & Significante \\
\hline
\end{tabular}

Con estos resultados, la hipótesis $\mathrm{H}_{2 c}$ es parcialmente soportada. Varias son las razones que explican el efecto moderador del liderazgo transformacional ya que, a pesar de que influencia al éxito de los proyectos, no necesariamente el mismo estilo se adapta a todos los tipos de proyecto, ni tampoco a todas las fases del ciclo de vida del mismo proyecto (Müller y Turner, 2007). El entorno donde se llevan a cabo los proyectos, y la realidad de cada organización en cuanto a estructura y cultura organizacional, podrían influenciar los resultados del estilo de liderazgo aplicado por el gerente de proyecto. 
a)

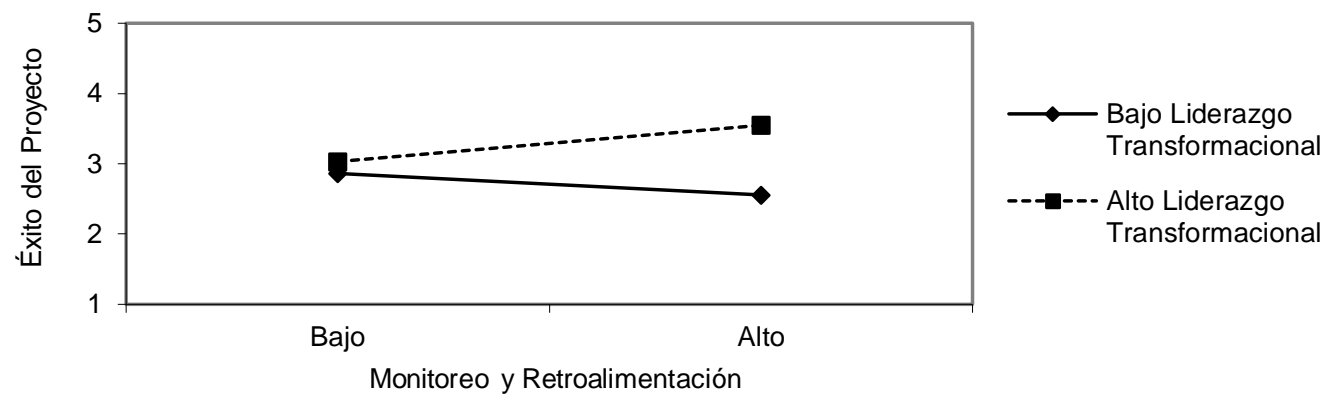

b)

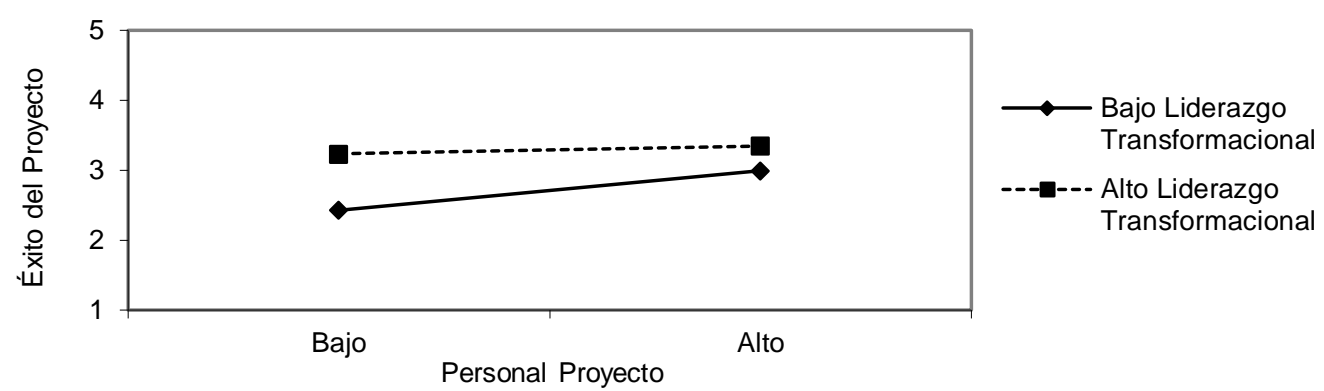

Fig. 4. Interacción: a) del monitoreo y retroalimentación y la experiencia; y b) del personal del proyecto y la experiencia

En esta etapa del análisis, se encontró que el liderazgo transformacional moderó a los siguientes factores críticos de éxito: (a) Monitoreo y Retroalimentación, hay una relación positiva entre este factor y el éxito del proyecto cuando el director del proyecto manifiesta tener un alto estilo de liderazgo transformacional (ver Figura 4a), y (b) Personal del Proyecto, independientemente si el nivel es bajo o alto, su relación con el éxito del proyecto es mayor para un alto nivel en el estilo de liderazgo transformacional del director de proyecto (ver Figura 4b).

A pesar de haber confirmado la influencia del estilo de liderazgo transformacional para el éxito del proyecto, los hallazgos sugieren que únicamente este estilo no es suficiente para moderar adecuadamente las relaciones entre los factores críticos de éxito y los criterios de éxito en los proyectos de TI, y sea necesario incorporar otros estilos adicionales de liderazgo al modelo, que combinados durante las fases del proyecto, expliquen en mayor medida su éxito.

\section{DISCUSIÓN FINAL}

Como contribución a la teoría, esta investigación ha obtenido resultados similares a los de Joseph y Marnewick (2017), a pesar de haberse efectuado en países en vías de desarrollo, caracterizados por una menor rigurosidad en la aplicación de modelos y un mayor empirismo en la valoración de la calidad de las decisiones. Los resultados obtenidos incrementan la validez de la moderación de la experiencia del director de proyecto en la relación entre los factores críticos de éxito y los criterios de éxito del proyecto. Otra contribución a la teoría es que se necesita considerar más variables para evaluar los factores que contribuyen con el éxito de un proyecto. Esto es de interés de la literatura, que alerta de solo tomar en cuenta al llamado triángulo de acero (tiempo, costo, y calidad) y que indica que deben utilizarse más criterios para para evaluar si un proyecto ha sido exitoso o no (Sánchez et al., 2017)

Respecto a la contribución a la práctica, este trabajo propone que la experiencia y la certificación son factores que modera la relación entre el entendimiento del cliente y el éxito de un proyecto. Por su parte, la experiencia contribuye a mejorar la capacidad de resolución de problemas, así como la certificación mejora el enfoque a contribuir con la misión del proyecto. Los mandos medios y altos en las organizaciones deberán tener presente la importancia de contar con directores de proyecto con suficiente experiencia, y certificados, para asegurar una buena relación con el cliente. Además, mantener un estilo de liderazgo transformacional contribuye a brindar retroalimentación al personal, así como a mejorar la relación con el equipo del proyecto.

Esta investigación puede ayudar a los directores de proyecto a un mejor entendimiento de la relación entre los factores críticos de éxito y los criterios de éxito del proyecto, y como el desarrollo de sus competencias puede potenciar este vínculo para incrementar la probabilidad de éxito de los proyectos bajo su gestión. Así mismo, las organizaciones deben concientizar que el rol de gerente de proyecto no sea una asignación temporal, sino el producto del desarrollo de competencias duras y blandas para asegurar que los resultados 
de los proyectos que necesita la organización, permitan alcanzar los beneficios esperados. Finalmente, para los institutos y asociaciones certificadoras en gestión de proyectos como el PMI, deben replantearse el esquema de proceso de certificación, con el fin de que los directores de proyectos que sean reconocidos con una certificación, demuestren que tal distinción realmente agrega valor a la gestión de proyectos y a las organizaciones, más allá de ser un simple requisito para ejercer el rol como director de proyecto.

Publicaciones relacionadas al estado de los proyectos se realizan continuamente en organizaciones a nivel mundial, para comprender las razones por las que los proyectos fracasan y su impacto en los resultados del negocio. La presente investigación contribuye con el conocimiento existente en gestión de proyectos, al encontrar mediante la aplicación de ecuaciones estructurales, los factores críticos de éxito que explican los criterios de éxito de los proyectos de TI, específicamente en Perú y Ecuador, al ser moderados por las competencias del gerente de proyecto. Para corroborar estos resultados, se recomienda llevar a cabo futuras investigaciones en otros países de la región para averiguar si el comportamiento puede generalizarse en América Latina.

\section{CONCLUSIONES}

De acuerdo a la investigación presentada y los resultados obtenidos, se plantean las siguientes conclusiones principales: 1) Los factores críticos de éxito del instrumento PIP que predicen los criterios de éxito del proyecto para el sector de TI en Perú y Ecuador son factores considerados como tácticos: comunicación, aceptación del cliente, el personal del proyecto, y la capacidad de resolución de problemas; 2) En este estudio, el personal del proyecto fue un factor que explico las dimensiones de éxito del proyecto, lo que reafirma la importancia de su gestión para el rendimiento del proyecto; 3) Con la inclusión de la experiencia y la certificación en el análisis, la comunicación fue poco significativa para el éxito del proyecto con una influencia negativa, lo que podría explicarse por la estructura de las organizaciones, el tipo de comunicación, y los canales de comunicación utilizados por el director del proyecto para distribuir la información a los diversos interesados en el proyecto, que no tuvieron el impacto esperado en el éxito del proyecto; 4) El factor crítico de éxito de la misión se volvió moderadamente significativo, influyendo positivamente en el éxito del proyecto, lo que reveló la importancia que el director del proyecto dio desde el principio al establecimiento de los objetivos y beneficios a alcanzar; 5) La experiencia no influyó en el éxito del proyecto, pero su inclusión moderó los factores críticos de éxito entendimiento al cliente, actividades técnicas, y la capacidad de resolver problemas; 6) La certificación fue significativa para el éxito del proyecto, y su inclusión moderó los factores críticos de éxito misión y entendimiento al cliente, sin embargo, por la relación negativa con el éxito del proyecto, los hallazgos sugieren que un director de proyecto certificado no necesariamente consigue resultados más exitosos respecto a un director de proyecto no certificado; 7) Se confirmó la influencia del estilo de liderazgo transformacional del director del proyecto en los criterios de éxito del proyecto y, a pesar de su impacto positivo e importante en el desempeño del proyecto, se puso de manifiesto que considerar sólo este estilo de liderazgo fue insuficiente para influir mejor en la relación entre los factores críticos de éxito y los criterios de éxito del proyecto; 8) Los resultados del presente estudio evidencian que al inicio del proyecto es importante establecer los objetivos y beneficios del proyecto a través de la misión, entender al cliente para poder obtener su aceptación al final del proyecto, contar con un equipo competente de proyecto con una alta capacidad de resolución de problemas durante la ejecución del proyecto, implementar una adecuada gestión de las comunicaciones, y mantener un monitoreo y retroalimentación constante durante todo el ciclo de vida del proyecto.

\section{REFERENCIAS}

Aga, D.A., Noorderhaven, N., y Vallejo, B., Transformational leadership and project success: The mediating role of teambuilding, https://doi.org/10.1016/j.ijproman.2016.02.012, Int. J. Proj. Manag., 34(5), 806-818 (2016)

Belout, A., y Gauvreau, C., Factors influencing project success: The impact of human resource management, https://doi.org/10.1016/s0263-7863(03)00003-6, Int. J. Proj. Manag., 22(1), 1-11 (2004)

Catanio, J., Armstrong, G., y Tucker, J., Project management certification and experience: The impact on the triple constraint, https://doi.org/10.4304/jait.4.1.8-19, J. Adv. Inf. Tech., 4(1) (2013)

Costantino, F., Di Gravio, G., y Nonino, F., Project selection in project portfolio management: An artificial neural network model based on critical success factors, https://doi.org/10.1016/j.ijproman.2015.07.003, Int. J. Proj. Manag., 33(8), 17441754 (2015)

Davis, K., Reconciling the views of project success: A multiple stakeholder model, https://doi.org/10.1177/8756972818786663, Proj. Manag. J., 49(5), 38-47 (2018)

Dörnyei, Z., Research methods in applied linguistics: Quantitative, qualitative, and mixed methodologies, Oxford University Press, New York, USA, (2007) 
Gemino, A., Reich, B.H., y Serrador, P.M., Agile, traditional, and hybrid approaches to project success: Is hybrid a poor second choice?, https://doi.org/ 10.1177/8756972820973082, Proj. Manag. J., 52(2), 161-175 (2021)

Hair, J.F., Black, W.C., Babin, B.J., y Anderson, R.E., Multivariate data analysis, 8a Ed., Cengage Publishers, Londres, Inglaterra, (2019)

Hoxha, L., y McMahan, C., Does a project manager's work experience help project success?, Int. J. Construc. Proj. Manag., ISSN: 1944-1436, 10(2), 155-172 (2018)

Jitpaiboon, T., Smith, S.M., y Gu, Q., Critical success factors affecting project performance: An analysis of tools, practices, and managerial support, https://doi.org/10.1177/8756972819833545, Proj. Manag. J., 50(3), $271-287$ (2019)

Joseph, N., y Marnewick, C., Investing in project management certification: Do organisations get their money's worth?, https://doi.org/10.1007/s10799-017-0275-y, Inf. Tech. Manag., 19(1), 51-74 (2017)

Medina, R., y Medina, A., The project manager and the organisation's long-term competence goal, https://doi.org/10.1016/j.ijproman.2014.02.011, Int. J. Proj. Manag., 32(8), 1459-1470 (2014)

Mir, F.A., y Pinnington, A.H., Exploring the value of project management: Linking project management performance and project success, https://doi.org/10.1016/j.ijproman.2013.05.012, Int. J. Proj. Manag., 32(2), 202-217 (2014)

Moradi, S., Kähkönen, K., y Aaltonen, K., From past to present - The development of project success research, https://doi.org/10.19255/JMPM02301, J. Mod. Proj. Manag., 8(1), 1-20 (2020)

Müller, R., y Jugdev, K., Critical success factors in projects: Pinto, Slevin, and Prescott - The Elucidation of Project Success, https://doi.org/10.1108/17538371211269040, Int. J. Managing Proj. Bus., 5(4), 757-775 (2012)

Müller, R., y Turner, R., The influence of project managers on project success criteria and project success by type of project, https://doi.org/10.1016/j.emj.2007.06.003, Europ. Manag. J., 25(4), 298-309 (2007)

Osei-Kyei, R., Chan, A.P.C., Javed, A.A., y Ameyaw, E.E., Critical success criteria for public-private partnership projects: International experts' opinion, https://doi.org/10.3846/1648715x.2016.1246388, Int. J. Strat. Prop. Manag., 21(1), 87-100 (2017)

Pinto, J.K., y Prescott, J.E., Variations in critical success factors over the stages in the project life cycle, https://doi.org/10.1177/014920638801400102, J. Manag., 14(1), 5-18 (1988)

Podsakoff, P.M., MacKenzie, S.B., Lee, J.-Y., y Podsakoff, N.P., Common method biases in behavioral research: A critical review of the literature and recommended remedies, https://doi.org/10.1037/0021-9010.88.5.879, J. Appl. Psych., 88(5), 879-903 (2003)

Ramos, P., Mota, C., y Corrêa, L., Exploring the management style of Brazilians project managers, https://doi.org/10.1016/j.ijproman.2016.03.002, Int. J. Proj. Manag., 34(6), 902-913 (2016)

Reinartz, W., Haenlein, M., y Henseler, J., An empirical comparison of the efficacy of covariance-based and variancebased SEM, https://doi.org/10.1016/j.ijresmar.2009.08.001, Int. J. Res. Market., 26(4), 332-344 (2009)

Sanchez, O.P., Terlizzi, M.A., y de Moraes, H.R., Cost and time project management success factors for information systems development projects, https://doi.org/10.1016/j.ijproman.2017.09.007, Int. J. Proj. Manag., 35(8), 1608-1626 (2017)

Savelsbergh, C.M., Havermans, L.A., y Storm, P., Development paths of project managers: What and how do project managers learn from their experiences?, https://doi.org/10.1016/j.jproman.2016.02.005, Int. J. Proj. Manag., 34(4), 559569 (2016)

Serrador, P., y Turner, R., The relationship between project success and project efficiency, https://doi.org/10.1002/pmj.21468, Proj. Manag. J., 46(1), 30-39 (2015)

Shenhar, A.J., y Dvir, D., Reinventing project management: The diamond approach to successful growth \& innovation, Harvard Business School Press, Boston, USA, (2007)

Shenhar, A.J., Dvir, D., Levy, O., y Maltz, A.C., Project success: A multidimensional strategic concept, https://doi.org/10.1016/s0024-6301(01)00097-8, Long Ran. Plann., 34(6), 699-725 (2001)

Slevin, D.P., y Pinto, J.K., The project implementation profile: New tool for project managers, Proj. Manag. J., 17(4), 5770 (1986)

Tyssen, A.K., Wald, A., y Spieth, P., The challenge of transactional and transformational leadership in projects, https://doi.org/10.1016/j.ijproman.2013.05.010, Int. J. Proj. Manag., 32(3), 365-375 (2014)

Wu, G., Liu, C., Zhao, X., y Zuo, J., Investigating the relationship between communication-conflict interaction and project success among construction project teams, https://doi.org/10.1016/j.ijproman.2017.08.006, Int. J. Proj. Manag., 35(8), 1466-1482 (2017)

Zhai, L., Xin, Y., y Cheng, C., Understanding the value of project management from a stakeholder's perspective: Case study of mega-project management, https://doi.org/10.1002/pmj.20099, Proj. Manag. J., 40(1), 99-109 (2009) 